\title{
Inhibition from semantically related primes: Evidence of a category-specific inhibition
}

\author{
TERESA A. BLAXTON and JAMES H. NEELY \\ Purdue University, West Lafayette, Indiana
}

\begin{abstract}
The phenomenon of inhibition from generating successive items within a category, reported by A. S. Brown (1981), was examined in two experiments. Subjects responded on target trials by either generating targets (e.g., generating BASS to B when it followed the category name FISH, Experiment 1) or reading them (reading BASS when it followed the category name FISH, Experiment 2). Prior to target trials, all subjects received priming trials consisting of either one or four exemplars from a single semantic category, which could be either the same category as the target's category (related priming condition) or an unrelated category (unrelated priming condition). In both experiments, different groups of subjects either read or generated primes. When primes were read, target response times (RTs) were always facilitated in the related priming condition compared with in the unrelated priming condition. However, when primes were generated, this facilitation from related primes was eliminated, except in the one-prime condition, when targets were also generated. When primes and targets were both generated, RTs in the related priming condition were slower following four primes than following one prime. Thus, category-specific inhibition from multiple related primes is greatest when both primes and targets must be actively retrieved.
\end{abstract}

Recent presentation of material semantically related to target information typically facilitates the processing or retrieval of that target information. For example, in a lexical (word-nonword) decision task, subjects are faster to respond that the letter string "nurse" is a word when it has been primed by a semantically related word such as "doctor" rather than by an unrelated word such as "butter" (Becker, 1979, 1980; Fischler, 1977; Meyer \& Schvaneveldt, 1971; Meyer, Schvaneveldt, \& Ruddy, 1975; Neely, 1976, 1977; Schvaneveldt \& Meyer, 1973). Facilitatory priming effects have also been observed in the Freedman-Loftus (1971) task in which subjects are given a category name and a letter (e.g., "animal-D") and are to produce a category exemplar beginning with that letter (e.g., "dog"). In this task, subjects are faster to respond if they have recently been tested on the current category than if they have not (Freedman \& E. F. Loftus, 1971; E. F. Loftus, 1973; G. R. Loftus \& E. F. Loftus,

This research was conducted in partial fulfillment of the requirements for a master of science degree at Purdue University for the first author and was supported by Grant R01 HD15054 awarded to the second author by the National Institute for Child Health and Human Development. We thank Kent Ross for assistance in data analyses and Lezlie DeGan, Linden Foster, Curt Gallenkamp, and Lisa Pelishek for helping test subjects. We also thank Howard Ranken, Henry Roediger III, and Richard Schweickert, who served on the master's committee, and who, along with David Payne, provided helpful criticisms and comments. Portions of this research were reported at the annual meeting of the Midwestern Psychological Association held in Chicago in May 1983. Requests for reprints should be sent to James H. Neely, Department of Psychological Sciences, Purdue University, West Lafayette, Indiana 47907.
1974). These facilitatory priming effects have typically been attributed to a dissipating wave of activation automatically spreading among related nodes in the memory network (e.g., Anderson, 1976; Collins \& E. F. Loftus, 1975; Posner \& Snyder, 1975; Schvaneveldt \& Meyer, 1973; but see also Lorch, 1982, Neely, 1977, p. 252, and Ratcliff \& McKoon, 1981).

Although most studies report that semantically related primes produce facilitation, some researchers have found that related primes may actually produce inhibition (see Roediger \& Neely, 1982, for a review). For example, J. Brown (1968) had an experimental group of subjects study a list of 25 states while a control group did light reading. In a subsequent test for the recall of all 50 states, the experimental group did better at recalling the previously presented 25 priming states but worse at recalling the 25 remaining target states than did the control group. Unfortunately, this inhibition effect is difficult to interpret for two reasons. First, because the control group did light reading, we have no way of knowing whether or not the prior study and/or retrieval of items from some category other than states would have produced as strong an inhibition effect as the semantically related state primes. This is problematic because, in episodic memory experiments, Roediger and Schmidt (1980) and A.D. Smith $(1971,1973)$ have shown that retrieval from one studied category can interfere with retrieval from some other studied category. Second, because the memory test was a free-recall test involving both priming and target items and because subjects probably recalled the priming items earlier than the target items in the recall period, we cannot easily ascertain 
the degree to which the impairment in recall of the target items was due to the prior study presentation of the semantically related primes or to the prior retrieval of those primes.

To address whether J. Brown's (1968) inhibition effect was due to the prior study or retrieval of the related primes, Karchmer and Winograd (1971) compared the inhibition obtained under free-recall instructions with that obtained when subjects were explicitly instructed to recall the presented priming items before recalling the nonpresented target items. Since the inhibition effect was slightly accentuated by explicit instructions to recall the priming items first, their results indicate that prior retrieval of the related priming items was contributing to the inhibition. However, it is still unclear if the prior retrieval of the priming items was the sole contributor to the inhibition effect or if the prior study of the related priming items was making a contribution to the inhibition effect beyond that of affecting the retrieval order of priming and target items.

A recent study by A. S. Brown (1981) indicated that prior retrieval of semantically related primes, without prior study of those primes, produces inhibition in the earlier described Freedman-Loftus paradigm employed by E. F. Loftus (1973). Contrary to Loftus's results, Brown found that the more prior tests there had been on a category, the slower subjects became in producing another exemplar of that category. To explain why he obtained inhibition from one prior test on a category rather than the facilitation that Loftus had obtained, Brown argued that, compared with Loftus's subjects, his subjects had covertly retrieved a larger number of a category's exemplars prior to the second test on that category. This is because Brown's subjects knew that they would be generating five successive items from the same category and were given a $5-\mathrm{sec}$ "warning" presentation of each new category name prior to the first categoryletter test probe for that category. Thus, during this 5 -sec warning presentation, Brown's subjects probably began to generate exemplars of that category in anticipation of potential upcoming restrictor letters. If so, Brown's subjects had already covertly retrieved several of a category's exemplars before the first category-letter test probe had even appeared for that category. Furthermore, as soon as they produced the correct answer to the first test on a category, Brown's subjects probably continued to retrieve additional exemplars of that category in anticipation of the second test probe for that category.

According to A. S. Brown (1981), then, it was the prior covert retrieval of several of a category's exemplars (during the warning presentation and after the correct response for the first test probe had been made) that produced retrieval inhibition on the second test of that category in his experiment. Since E. F. Loftus's (1973) subjects did not receive a warning presentation of a category name and only occasionally received two consecutive tests on the same category, they could not predict which category would be tested on the upcoming trial. Thus, they probably did not covertly try to retrieve additional exemplars of a category once they had overtly produced the correct response for that category's test probe. Consequently, retrieval interference from such additional prior retrievals would not have been present on the second test of a category to cancel out the facilitation produced by the prior test on that category in Loftus's experiment. ${ }^{1}$

A. S. Brown's (1981) Experiments 2 and 3 indirectly support the foregoing analysis. In these experiments, Brown presented several series of eight pictures from the same semantic category, and the subjects' task was to name each picture aloud as it appeared. Since this was such an easy task, subjects probably did not covertly try to generate category exemplars in anticipation of the upcoming picture stimulus, with the result that Brown's picture-naming data replicated E.F. Loftus's (1973) finding of facilitation from one recent test on the target's category. However, as the names of more and more additional pictures from the same category were retrieved on target trials, response times (RTs) became slower, indicating the eventual buildup of interference produced by several prior retrievals of items belonging to the same semantic category as the target item.

\section{EXPERIMENT 1}

If A. S. Brown's (1981) analysis was correct, under conditions in which subjects do not covertly retrieve exemplars either prior to or between probe trials in the Freedman-Loftus task, one should be able to obtain both a facilitatory priming effect from one prior test on a category and an inhibitory priming effect from several prior tests on a category. Experiment 1 tested this hypothesis by employing two procedures that were expected to minimize covert retrievals before and between probe trials. First, the 5-sec "warning" presentation of a category name that A. S. Brown (1981) used was eliminated. Second, subjects did not always make the same number of consecutive retrievals from a particular category before being tested on another category. That is, as in E. F. Loftus's (1973) experiment, subjects overtly retrieved the correct answer for the current trial and then had to wait for the beginning of the next trial before knowing which category would be tested.

A. S. Brown's (1981) procedures were further modified to permit a more detailed analysis of any inhibition effects that might be obtained. Although Brown demonstrated that RTs increased as the number of retrievals from the target's category increased, he did not have the proper controls for demonstrating that this increase in RTs was due to a buildup of category-specific inhibition. That is, since Brown did not test an unrelated priming condition, it may be that the increase in RTs that he observed could also have been produced by several prior retrievals from a single semantic category unrelated to the target's category. ${ }^{2}$ Experiment 1 was expected to help clarify this issue, since it would permit a comparison of target retrieval times obtained when the target 
probe followed one or four consecutive tests either on the same category as the target category (the related priming condition) or on some other category (the unrelated priming condition). If the increase in RTs produced by four primes as compared with one prime was greater in the related priming condition than in the unrelated priming condition, the results would favor a category-specific inhibition interpretation of $\mathrm{A}$. S. Brown's (1981, Experiment 1) results. If, on the other hand, the increase in RTs from the unrelated one-prime to four-prime condition was equal to that observed for the related one-prime to four-prime condition, a nonspecific-inhibition interpretation would be favored. Finally, if the increase in RTs from the unrelated oneprime to four-prime condition was greater than that observed for the related one-prime to four-prime condition, one would conclude that there was a nonspecific inhibition effect that was being partially offset by the summation of facilitative spreading activation in the related four-prime condition.

Experiment 1 also tested A. S. Brown's (1981) hypothesis that active retrieval of related primes is necessary for inhibition to occur. To evaluate this hypothesis, a read-generate group, in which subjects read primes aloud and generated category members only on target trials, was tested. If active retrieval of related primes was necessary for producing inhibition, facilitation was expected to occur in the related four-prime condition in this read-generate group. Facilitation, rather than inhibition, was expected because, unlike in the generategenerate group, there would be no active-retrieval inhibition accumulating on the four related priming trials to offset the facilitatory effects of spreading activation.

In summary, then, if active retrieval of related primes was necessary for inhibition to accrue, RTs in the related priming condition were expected to be faster than those in the unrelated priming condition in both the one-prime and the four-prime conditions in the read-generate group, since there would be no active retrievals on priming trials to produce an offsetting inhibition effect in the related four-prime condition in this group. In the generategenerate group, on the other hand, a different pattern of data was expected to emerge. Specifically, since the current procedures made it unlikely that subjects would be covertly retrieving category exemplars before and between probe trials, retrieval times in the related oneprime condition were expected to be faster than those in the unrelated one-prime condition, thereby replicating E. F. Loftus's (1973) facilitation effect. However, after four consecutive active retrievals from the target item's category, inhibition was expected to have accrued such that retrieval times in the related four-prime condition would be slower than in the related one-prime condition, thereby replicating A. S. Brown's (1981) inhibition effect. Finally, if the increase in RTs in the related fourprime condition as compared with the related one-prime condition was not accompanied by a similar increase across the corresponding unrelated priming conditions, category-specific inhibition would be demonstrated.

\section{Method}

Subjects. Of the 144 subjects tested in the experiment, 134 participated to fulfill an introductory psychology course requirement. The remaining 10 subjects were paid $\$ 3.00$ for their participation. Seventy-two subjects were assigned randomly to the read-generate group and 72 to the generate-generate group.

Design. Prior to generating a target item, each subject was exposed to one of four priming conditions formed by crossing one or four primes with two different types of primes-related or unrelated. The one between-subjects variable was whether the priming items had been generated (the generate-generate group) or read (the read-generate group) prior to the generation of the target item. Thus, the design was a 2 (group: generate-generate vs. read-generate) $\times 2$ (prime type: related vs. unrelated) $\times 2$ (number of primes: one vs. four) mixed-factor design.

In addition to the related and unrelated priming conditions, task-shift control conditions consisting of one or four primes were also included in each group. The main reason for including these conditions was to help disambiguate the interpretation of a possible main effect of group. Specifically, a main effect of group could be due either to differences in the retrieval tasks performed on priming trials in the generate-generate and the read-generate groups or to the fact that subjects in the readgenerate group shifted from a reading task on priming trials to a generation task on target trials, whereas subjects in the generategenerate group always performed a generation task on both priming and target trials.

To help partial out any effect due to task shifting in the readgenerate group, one-prime and four-prime task-shift control conditions were included. These task-shift control conditions required that subjects switch tasks from reading items on priming trials to generating items on target trials, while at the same time not exposing the subjects to a new set of priming items. This was accomplished by having target trials in the task-shift control condition in the read-generate group always be primed by "ONE-A" in the one-prime case and by "ONE-A," "TWO-B," "THREE-C," and "FOUR-D," in that order, in the four-prime case. In the generate-generate group, the task-shift control conditions were similar to those in the read-generate group, in that the subjects did not receive any new priming items, but different from them in that the subjects did not shift tasks from reading items on priming trials to generating items on target trials. Rather, they generated items on both priming and target trials. This was accomplished by having subjects in the generate-generate group see an " $X$ " on the screen on task-shift control priming trials instead of the letters " $A$," "B," "C," or " $D$ " that the subjects in the read-generate group saw and read. As soon as the subjects in the generate-generate group saw the " $X$," they were to generate " $A$," " $B$," "C," or " $D$," respectively, depending on the immediately preceding number, "ONE," "TWO," "THREE," or "FOUR."

The data from these task-shift control conditions can be used to interpret a main effect of group for the related and unrelated priming conditions, as follows. A main effect of group for the related and unrelated priming conditions equivalent to that in the task-shift control conditions would suggest that this group effect is due solely to differences in the task-shifting requirements for the read-generate group relative to the generate-generate group. On the other hand, a main effect of group in the related and unrelated priming conditions different from that in the task-shift control conditions would suggest that the effect of group in the related and unrelated priming conditions was due, at least in part, to the effects of reading versus generating as primes new exemplars from a single category.

Materials. One hundred and nineteen semantic categories were selected from the Battig and Montague (1969), Hunt and Hodge (1971), and Shapiro and Palermo (1970) category norms. Five dominant exemplars from each category were used, with no two exemplars from any one category beginning with the same letter. One category, SPORTS, occurred in both base lists, so that, in effect, 120 categories were employed.

Two base lists of stimulus materials, A and B, each contained 
60 category names and the category names' five respective exemplars. The target item from each category was the most dominant exemplar of that category, unless the most dominant category exemplar shared the same initial letter with another highly dominant exemplar, for example, "baseball" and "basketball" for the category SPORTS. In such a case, a less ambiguous but highly dominant category member was chosen as the target. The four exemplars of a category not used as the target served as related primes for that category, so that target items were never used as primes for any category.

Categories for each base list were always presented in the same order, with the two base lists constructed such that categories in corresponding positions in the $A$ and $B$ versions were semantically unrelated to one another. Thus, when a category in a given position in one base list was to serve in an unrelated priming condition, the unrelated primes could be obtained by using the related primes for the corresponding category of the other base list. Within the related priming condition and within the unrelated priming condition, the very same prime always immediately preceded retrieval of the target in the one-prime and the four-prime conditions.

In constructing one complete base list, a random order of the six [3 types of primes (related, unrelated, and task-shift control) $x 2$ (numbers of primes)] priming conditions was adopted, and then repeated 10 times to accommodate the 60 critical target trials making up that list. Five other stimulus lists were constructed from each base list, such that, across lists, each target item was presented once in each of the six priming conditions. Thus, altogether, 12 lists were constructed, with 6 being composed of targets from base list $A$ and 6 of targets from base list B. Each subject was tested on only 1 of the 12 lists, with no primes or targets ever being repeated in a particular list.

Procedure. Individually tested subjects were given instructions and practice with each type of prime before the experimental session began. The timing on priming and target trials was the same. Thus, the target trials were in no way temporally distinct from the priming trials, and the priming trials that followed a target were not temporally distinct from the priming trials that followed other priming trials. On related and unrelated priming trials, the subjects in the read-generate group saw alternating displays of a category name followed by a member of the category. Each category name was displayed alone for $2 \mathrm{sec}$, and then the prime was displayed alone for $8 \mathrm{sec}$. As subjects saw a category name or exemplar, they read it aloud. On task-shift priming trials, the same procedure was adopted with the number names (ONE, TWO, THREE, or FOUR) replacing the category names and the letters (A, B, C, or D) replacing the exemplar primes. On target trials, the subjects in the read-generate group saw a category name displayed for $2 \mathrm{sec}$ and then an 8-sec single-letter display during which they were to respond by saying aloud a category member beginning with that letter. The subjects in the generate-generate group followed the same general procedure as those in the read-generate group, except that they were asked to generate a category member from a letter cue on the related and unrelated priming trials and had to "generate" the "A," "B," " $C$," or " $D$ " response from the " $X$ " cue on the task-shift control priming trials. Whenever a subject did not produce an appropriate response on either a prime or a target trial in the generategenerate group, the experimenter said the response aloud at the end of the response period. This ensured that all subjects in the generate-generate group received a prime on all trials.

In each $45-\mathrm{min}$ session, a subject was tested on 60 target trials-10 target trials in each of the six priming conditions. Stimuli were produced by a Flashwriter board and were presented visually on a Sony (Model CVM-131) video monitor. RTs on target trials were measured to the nearest millisecond and were timed from the onset of the restrictor letter to the closure of a Lafayette Instruments voice-activated relay (Model 18010). The voice-activated relay was closed by the subject's vocal response into a Telex CS-91 microphone mounted on a headset. Timing and data storage were controlled by a Cromemco Z-80 microcomputer system.

\section{Results}

Statistical analyses of the data took the following form. First, a 2 (group: generate-generate vs. readgenerate) $\times 2$ (prime type: related vs. unrelated) $\times 2$ (number of primes: one vs. four) analysis of variance (ANOVA) was performed on the dependent measure of interest. If this ANOVA revealed that group and some other factor(s) yielded a statistically significant interaction, the data for each group were submitted to separate 2 (prime type) $\times 2$ (number of primes) ANOVAs. (A similar procedure was followed for the task-shift control data, except that type of prime was not a factor in the ANOVAs.) The statistical reliability of individual post hoc comparisons within a group was evaluated with Fisher's least significant difference (LSD) test, the error term for which was based on the mean squared error (MSe) for the prime type $\mathrm{x}$ number of primes interaction. Unless otherwise indicated, all significance levels were set at $\mathrm{p}<.05$ (two-tailed in the case of the LSD test).

Unexpected responses and response omissions. Although the stimulus pair "FISH-B" was intended to elicit the response "bass," subjects sometimes gave responses such as "barracuda" or "bluegill." RTs for such trials were omitted from the statistical analyses because, in certain priming conditions, subjects might be prone to generate unusual, low-frequency target items. Although this effect would be interesting, it would artificially inflate target RTs in that condition. To make a strong case for inhibition from related primes, one must compare RTs for exactly the same (i.e., experimenter-defined) targets for the related and unrelated one-prime and fourprime conditions.

The mean percentages of unexpected target responses (and target response omissions) for each priming condition are presented in Table 1. The ANOVA that included group as a factor indicated that the only statistically significant effect for the mean percentage of trials on which

Table 1

Mean Percentages of Unexpected Responses (UR) and Response Omissions (RO) for the Generate-Generate and Read-Generate Groups

\begin{tabular}{|c|c|c|c|c|}
\hline \multirow{3}{*}{$\begin{array}{l}\text { Number of } \\
\text { Primes }\end{array}$} & \multicolumn{4}{|c|}{ Prime Type } \\
\hline & \multicolumn{2}{|c|}{ Related } & \multicolumn{2}{|c|}{ Unrelated } \\
\hline & UR & RO & UR & RO \\
\hline & \multicolumn{4}{|c|}{ Generate-Generate Group } \\
\hline 1 & 9.9 & 10.3 & 10.3 & 7.5 \\
\hline \multirow[t]{2}{*}{4} & 8.2 & 11.2 & 10.3 & 9.2 \\
\hline & \multicolumn{4}{|c|}{ Read-Generate Group } \\
\hline 1 & 7.1 & 6.0 & 7.9 & 5.0 \\
\hline 4 & 6.9 & 6.9 & 11.1 & 5.8 \\
\hline
\end{tabular}


subjects gave a correct response other than the experimenter-defined target was the main effect of prime type $[F(1,142)=4.13$, MSe $=122.45]$, with fewer unexpected responses given in the related priming condition $(8.0 \%)$ than in the unrelated priming condition $(9.9 \%)$. Of greater importance, however, is the fact that the group $\mathrm{x}$ prime type $\mathrm{x}$ number of primes interaction was not reliable $[\mathrm{F}(1,142)<1, \mathrm{MSe}=78.87]$.

Those trials for which subjects failed to emit a target response during the 8 -sec response period were, like the unexpected responses, excluded from the RT analyses. For response omissions, the ANOVA that included group as a factor yielded only two statistically significant effects. The subjects in the generate-generate group had more response omissions $(9.5 \%)$ than those in the read-generate group $(5.9 \%)[\mathrm{F}(1,142)=11.73$, MSe $=$ $160.13\}$, and the response omission rate was higher in the related priming condition $(8.6 \%)$ than in the unrelated priming condition $(6.9 \%)[\mathrm{F}(1,142)=5.07$, MSe $=$ $85.54]$. Once again, the group $x$ prime type $x$ number of primes interaction did not approach significance $[\mathrm{F}(1,142)<1, \mathrm{MSe}=65.08]$.

As for the task-shift control conditions, an ANOVA performed on the unexpected response data did not reveal any significant main effects or interactions. (The mean percentage of unexpected responses was $10.4 \%$.) However, for response omissions, subjects in the generategenerate group failed to respond on more task-shift control target trials $(8.2 \%)$ than did subjects in the readgenerate group $(4.9 \%)[\mathrm{F}(1,142)=7.55, \mathrm{MSe}=110.38]$, with no other effects or interactions being significant.

Response times. Since the RT distributions were positively skewed, the median RT for each subject in each priming condition was used in the statistical analyses. The means of the median RTs are displayed in Figure 1. The first notable aspect of these data is that in the readgenerate group (see right panel of Figure 1), the typically observed facilitation effect was obtained in the related priming condition relative to the unrelated priming con-

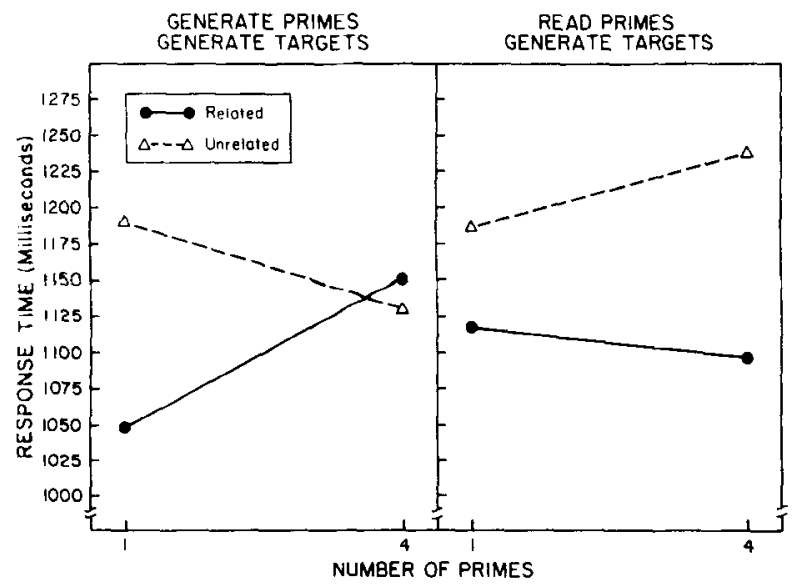

Figure 1. Means of the median RTs as a function of prime type and number of primes for Experiment 1. dition for both the one-prime and the four-prime conditions. Thus, when active retrieval of the primes was not required, facilitation was observed in the related priming condition relative to the unrelated priming condition in both the one-prime and the four-prime conditions. However, as can be seen in the left panel of Fig. ure 1 , the pattern of data was quite different in the generate-generate group, in which subjects were required to retrieve the primes actively. Although RTs in the related one-prime condition in the generate-generate group were considerably faster than those in the unrelated oneprime condition, thus replicating E. F. Loftus's (1973) facilitation effect, there was no evidence of facilitation in the related four-prime condition relative to the unrelated four-prime condition. Indeed, RTs in the related four-prime condition were slightly slower than those in the unrelated four-prime condition. Furthermore, as A. S. Brown (1981) found, RTs in the related fourprime condition were considerably slower than those in the related one-prime condition. Since this increase in RTs in the related four-prime condition relative to the related one-prime condition was not accompanied by a similar increase in the unrelated four-prime condition relative to the unrelated one-prime condition, the present data indicate that the inhibition effect is indeed category specific.

These general observations were supported by the statistical analyses. The ANOVA that included group as a factor revealed that the group $\mathrm{x}$ prime type $\mathrm{x}$ number of primes interaction was indeed significant $[F(1,142)=$ $5.53, \mathrm{MSe}=91,263.71]$. For the read-generate group, the $108-\mathrm{msec}$ facilitation effect in the related priming conditions relative to the unrelated priming conditions was significant $[\mathrm{F}(1,71)=10.50, \mathrm{MSe}=79,486.63]$ and was of statistically comparable magnitudes in the one-prime and the four-prime conditions $[\mathrm{F}(1,71)=$ $1.16, \mathrm{MSe}=94,785.37$, for the prime type $\mathrm{x}$ number of primes interaction]. As for the generate-generate group, the prime type $\mathrm{x}$ number of primes interaction was significant $[F(1,71)=5.14, \mathrm{MSe}=87,388.77]$. With an LSD of $84 \mathrm{msec}$, the $139-\mathrm{msec}$ facilitation effect in the related one-prime condition compared with the unrelated one-prime condition was significant, as was the $101-\mathrm{msec}$ inhibition effect in the related four-prime condition compared with the related one-prime condition. However, neither the 57-msec decrease in RTs in the unrelated four-prime condition relative to the unrelated one-prime condition nor the 19-msec inhibition effect in the related four-prime condition relative to the unrelated four-prime condition was significant.

As for the task-shift control data, the mean RTs in the task-shift control conditions in the read-generate group were 1,163 and $1,186 \mathrm{msec}$ for the one-prime and the four-prime conditions, respectively, and in the generate-generate group, they were 1,123 and 1,064 msec for the one-prime and the four-prime conditions, respectively. Although RTs were $81 \mathrm{msec}$ slower in the read-generate group than in the generate-generate group, 
thereby implicating a general performance deficit when subjects had to shift from reading to generating, this between-subject effect was not significant $[F(1,142)=$ $2.67, \mathrm{MSe}=178,254.4]$. No other main effect or interaction reached conventional levels of statistical significance for the task-shift control conditions.

\section{Discussion}

One important feature of the data of Experiment 1 concerns evidence of facilitation from related primes in both the generate-generate and the read-generate groups. The finding of faster RTs in the related one-prime condition than in the unrelated one-prime condition when subjects actively generated primes in the generategenerate group replicates E. F. Loftus's (1973) work. Although this facilitation from related primes did not occur in the four-prime condition when subjects actively generated primes, it occurred in both the one-prime and the four-prime conditions when the subjects merely read primes in the read-generate group.

In addition to replicating E. F. Loftus's (1973) facilitation effect, the results from our generate-generate group confirm A. S. Brown's (1981) inhibitory semantic priming effect as well. That is, in our generate-generate group, as in Brown's Experiment 1, RTs in the related priming condition increased as the number of primes increased. Since RTs in the unrelated priming condition did not show a similar increase (in fact, they decreased slightly), our data provide stronger support for categoryspecific inhibition than do Brown's data. A further source of evidence for inhibition was that the facilitation observed in the related four-prime condition in the readgenerate group was completely eliminated when the subjects were required to retrieve primes actively in the generate-generate group, with this loss of facilitation being due to a slowing in RTs in the related four-prime condition relative to those in the related one-prime condition.

A. S. Brown, Bean, and Whiteman (Note 1) conducted two experiments with conditions analogous to ours and obtained inhibition effects strikingly similar to ours. In Brown et al,'s Experiment 2, prior to naming a picture, subjects generated either zero, one, or three category items from word fragments that were category exemplars from which all vowels had been omitted (e.g., $\mathrm{T}_{\text {_B }} \mathrm{BL}$ _ for the category FURNITURE). These category prime words were strongly semantically related to the picture's category, weakly related to the picture's category, or from some other unrelated semantic category. As in our generate-generate group, Brown et al. observed that, as the number of primes increased from zero to three, naming latencies for the target pictures in the strongly related priming condition increased, whereas those for the unrelated priming condition decreased. (Latencies in the weakly related priming condition increased nonsignificantly.) Thus, their data, as do ours, provide evidence for category-specific inhibition in that target RTs increased as the number of active retrievals of highly related primes increased but not as the number of active retrievals of unrelated primes increased. (Unlike in our generate-generate data, in Brown et al.'s data there was no evidence of facilitation from one strongly related prime relative to one unrelated prime. We will return to this point in discussing the results from our Experiment 2.)

\section{EXPERIMENT 2}

Although the data from Experiment 1 showed that a category-specific inhibitory priming effect occurred only when subjects actively generated primes, two issues remained unresolved. One was whether active retrieval of target, as well as of priming, information is necessary for obtaining category-specific inhibition. If such inhibition were to occur in a generate-read group in which subjects actively retrieved priming items but only read target items, it would be established that active retrieval on target trials is not necessary for obtaining category-specific inhibition. A second issue was whether the categoryspecific inhibition effect obtained in the generategenerate group depends on subjects' actively retrieving both primes and targets or is simply a function of subjects' performing the same task on primes and targets. If category-specific inhibition depends on there being an active retrieval of both primes and targets, inhibition should not occur in a read-read group, in which subjects read both primes and targets. If, on the other hand, category-specific inhibition depends on subjects' performing the same task on the semantically related primes and targets, category-specific inhibition should occur in a read-read group. Experiment 2 examined these possibilities.

\section{Method}

The design, materials, and procedures were identical to those of Experiment 1, with the exception that the subjects always read, rather than generated, the targets. The read-read and generate-read groups each consisted of 72 introductory psychology students who participated in partial fulfillment of a course requirement and had not participated in Experiment 1 .

\section{Results}

The data analyses were of the same form as those used in Experiment 1. Since targets were always read, there were no response failures or unexpected responses. The means of the median RTs for all priming conditions are presented in Figure 2. As shown in the right panel, the typical facilitation in the related priming conditions relative to the unrelated priming conditions was observed in the read-read group, as had been the case in the read-generate group in Experiment 1. However, as may be seen in the left panel of Figure 2, this facilitation ef fect was completely eliminated when subjects generated primes prior to reading targets in the generate-read group. Although the absence of facilitation in the generateread group provides some evidence for inhibition from the generation of related primes, unlike in the generategenerate group in Experiment 1, the increase in response times in the related four-prime condition relative to the 
related one-prime condition was not greater than that observed in the corresponding unrelated priming conditions. Finally, RTs in the generate-read group were generally much slower than those in the read-read group.

These differing patterns of data for the read-read and generate-read groups were borne out by a significant group $\times$ prime type interaction $[\mathrm{F}(1,142)=4.22$, MSe $=$ $5,736.04]$ and a marginally reliable group $x$ number of primes interaction $[\mathrm{F}(1,142)=3.85$, MSe $=2,303.94$, $\mathrm{p}<.06]$. Also, the main effect of group was significant $[F(1,142)=140.10, \mathrm{MSe}=31,520.86]$, with RTs in the generate-read group being 175 msec slower than those in the read-read group. However, unlike in Experiment 1, the group $\mathrm{x}$ prime type $\mathrm{x}$ number of primes interaction did not approach significance $[F(1,142)<1$, MSe = $2,611,02]$. Separate ANOVAs performed for the two groups revealed that in the read-read group the $25-\mathrm{msec}$ facilitation in the related priming conditions compared with the unrelated priming conditions was highly significant $[\mathrm{F}(1,71)=15.58, \mathrm{MSe}=2,984.70]$. Although the 0 -msec "facilitation" effect in the generate-read group was obviously not significant, the main effect of number of primes was significant $[\mathrm{F}(1,71)=4.93$, MSe $=$ $3,290.59$ ], with RTs following four primes being $15 \mathrm{msec}$ slower than those following one prime. Unlike in the generate-generate group of Experiment 1, the prime type $\mathrm{x}$ number of primes interaction was not reliable in either the generate-read or the read-read group (both $\mathrm{Fs}_{\mathrm{s}}<1$ ).

As for the task-shift control conditions, the mean RTs in the read-read group were 553 and $544 \mathrm{msec}$ for the one-prime and the four-prime conditions, respectively, and those in the generate-read group were 696 and $709 \mathrm{msec}$ for the one-prime and the four-prime conditions, respectively. The overall ANOVA showed that only the main effect of group was significant $[F(1,142)$ $=81.54, \mathrm{MSe}=20,831.23]$, reflecting the fact that target RTs in the task-shift control conditions were

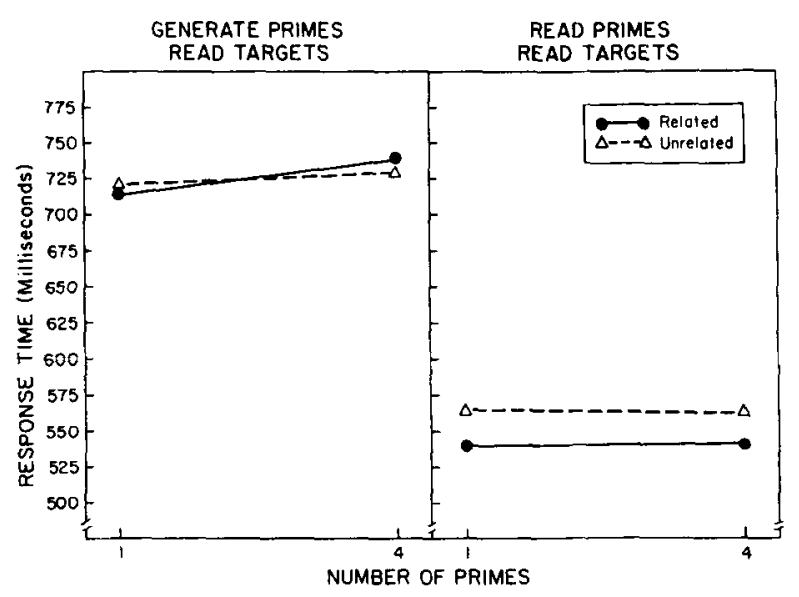

Figure 2. Means of the median RTs as a function of prime type and number of primes for Experiment 2.
$154 \mathrm{msec}$ slower in the generate-read group than in the read-read group. Since this 154-msec effect is comparable to the 175-msec effect obtained for the related and the unrelated priming conditions and since read-generate RTs in Experiment 1 were slower than generate-generate RTs in the task-shift control conditions (although not significantly so), we conclude that this general nonspecific interference resulted from subjects' shifting tasks from priming trials to target trials and not from a greater interference from generating primes compared with reading primes.

\section{Discussion}

Taken together, the results of Experiments 1 and 2 help to delineate the variables that control category. specific inhibition. Specifically, evidence of inhibition in the form of slower RTs following four related primes than following one related prime was obtained only in the generate-generate group, in which subjects generated both primes and targets. The fact that no strong inhibition effects were obtained in the read-generate and the generate-read groups suggests that the category-specific inhibition obtained in the related four-prime condition in the generate-generate group was due either (1) to subjects' actively retrieving items on both the priming and the target trials or (2) to subjects' performing the same task on the priming and the target trials. The absence of inhibition in the read-read group clearly showed that it was the active retrieval of both primes and targets that produced the category-specific inhibition in the generategenerate group. Also, since inhibition accrued when subjects named both priming and target pictures in A.S. Brown's (1981) experiments but not when they named both priming and target words in our read-read group, it seems that picture naming must involve a more active retrieval process than does word naming (see Potter \& Faulconer, 1975).

One aspect of the results obtained in Experiment 2 was unexpected. The absence of facilitation from one related prime relative to one unrelated prime in the generate-read group runs counter to the facilitation effects observed by E. F. Loftus (1973) and to those observed in the other three groups tested in the present experiments. Interestingly, A.S. Brown et al. (Note 1) also failed to obtain a facilitation effect from the presentation of one strongly semantically related prime compared with the presentation of one unrelated prime in a task analogous to that performed by the subjects in our generate-read group. In Brown et al.'s task, subjects actively generated category exemplars from word frag. ments on priming trials and then named a picture on target trials. Similarly, subjects in our generate-read group actively generated category exemplars on priming trials and merely read words on target trials. If one considers picture naming and reading to involve less active retrieval than generating a word from a word fragment, Brown et al.'s and our results converge on the conclusion that facilitation from one related prime will not be obtained 
if the amount of active retrieval on priming trials is greater than that on target trials. Rather, facilitation will be obtained only if the amount of active retrieval on priming trials is less than that required on target trials (as in our read-generate group) or equal to that required on target trials (as in our read-read and generate-generate groups, A. S. Brown's, 1981, picture-naming/picturenaming condition, and E. F. Loftus's, 1973, task). Of course, without additional research, these conclusions about how facilitation from one related prime depends on the relative difficulties of the tasks performed on primes and targets must remain speculative. (However, as we shall discuss shortly, when we consider results reported by M. C. Smith, Theodor, \& Franklin, 1983, this conclusion seems to hold only when subjects must retrieve the names of the primes and targets.)

\section{GENERAL DISCUSSION}

The present research showed that semantically related primes may either facilitate or inhibit the retrieval of tar. get information, depending on the retrieval demands of the tasks performed on the primes and targets. When the subjects read primes, they were faster to read or generate targets following related primes than following unrelated primes, regardless of whether there was one prime or four primes. This facilitation is similar to that obtained in the Freedman-Loftus paradigm (e.g., E. F. Loftus, 1973) and in lexical-decision and word-naming tasks (e.g., Meyer, Schvaneveldt, \& Ruddy, 1975). However, when subjects generated primes, there were two sources of evidence of inhibition from related primes. First, the facilitation from related primes that occurred when subjects read primes was eliminated when primes were generated. This was so for the one-prime and the four-prime conditions in the generate-read group and for the fourprime condition in the generate-generate group. A second, stronger source of evidence for inhibition from related primes occurred in the generate-generate group, in which RTs were slower following four related primes than following one related prime. This evidence for inhibition supports A.S. Brown's (1981) conclusion that self-generated primes related to the target inhibit the retrieval of that target.

When inferring inhibition from related primes from the present data, we appeal to the operation of an inhibitory mechanism that is invoked when subjects actively generate related primes. This inhibitory mechanism competes with the operation of a facilitatory mechanism and cancels out the facilitation effect that normally occurs when subjects merely read related primes. Because the present data provide no direct evidence for the independent existence of these countervailing processes of facilitation and inhibition, our conclusion that inhibition occurred should be accepted only tentatively until such evidence becomes available. Indeed, one could explain the elimination of facilitation from related primes by simply assuming that facilitation from related primes decays more rapidly when primes are generated rather than read. Although this "decay of facilitation" hypothesis can, in principle, explain most of the data from the present studies, it seems highly unlikely that facilitation would decay more rapidly when primes are generated rather than read. In fact, a result reported by M. C. Smith et al. (1983, Experiment 1) suggests that more facilitation is obtained as primes are processed to a deeper level (see also Henik, Friedrich, \& Kellogg, 1983; Irwin \& Lupker, 1983; Parkin, 1979; M. C. Smith, 1979). Such a finding runs counter to the results we obtained in the generate-read group in Experiment 2. That is, if deeper processing of primes leads to greater facilitation and if generation requires deeper processing than reading, one would have expected greater facilitation following one related prime in the generate-read group than in the read-read group, rather than the complete absence of facilitation that we obtained. One possible explanation for this discrepancy between M. C. Smith et al.'s and our results is that in M. C. Smith et al.'s study, subjects were always given the priming word and asked to perform some operation on it, whereas in our generate-read group, subjects had to produce the primes themselves. Thus, there may be fundamental differences between the processes involved in generating a prime given a single letter as opposed to making some decision about that prime once it has been provided.

Despite the discrepancy between M. C. Smith et al.'s and our findings, both sets of results converge on the conclusion that the types of operations performed on primes and targets play a key role in determining whether facilitatory or inhibitory priming effects will be obtained in semantic memory paradigms. Thus, in one sense, facilitation effects from related primes are not automatic, as some have assumed (e.g., Neely, 1977; Posner \& Snyder, 1975), nor are inhibition effects from related primes automatic, as others have argued (Brown, 1979; but see also Roediger, Neely, \& Blaxton, 1983). Rather, facilitation is not obtained unless presented primes are processed to a relatively deep level, and inhibition occurs only when several related primes are actively retrieved by the subjects themselves. ${ }^{3}$

Now that the conditions under which inhibition from related primes occurs are better understood, there are several general theoretical approaches that may be offered to account for this inhibition. One of these (see A.S. Brown, 1981) is embodied in the search-ofassociative-memory (SAM) model proposed by Raaijmakers and Shiffrin (1981). In SAM, probe cues are used to effect retrieval, and the retrieval of an item strengthens its association to these probe cues. Thus, the recurring retrieval of previously retrieved and strengthened priming items can "block" the retrieval of targets (see J. Brown, 1968, and Rundus, 1973). This retrievalblockage explanation can account for Brown's and our results if one makes the following reasonable assumptions: (1) The category-name probe restricts retrieval to 
items from that category so that previously retrieved priming items from some category other than the target item's category will not block retrieval of the target. (2) The letter probe is used to check the correctness of a retrieved item but does not restrict retrieval to items beginning only with that letter (see Roediger \& Tulving, 1979). If this is not assumed, previously retrieved category members beginning with a letter other than the target's first letter could not block retrieval of the target.) (3) When subjects generate a prime, they implicitly retrieve more items from the prime's category than if they read that prime. Thus, when primes are generated rather than read, there will be a greater number of previously retrieved primes from the target item's category to block the target's retrieval. (This assumption is the one made by A. S. Brown, 1981.) Clearly, then, SAM has the machinery to account for inhibition effects.

In addition to being able to account for inhibitory semantic priming effects in semantic memory paradigms, SAM can also account for inhibition from semantically related cues in episodic recall tasks (e.g., Mueller \& Watkins, 1977; Roediger, 1973; Rundus, 1973; Watkins, 1975). However, applying SAM to episodic recall tasks, Raaijmakers and Shiffrin (1981) chose to appeal to mechanisms completely different from those we used above to explain inhibitory semantic priming effects in semantic memory. Specifically, they assumed that subjects in an episodic memory task form both context-to-item and item-to-item associations when memorizing a categorized list of words. Rehearsal time on specific items produces a positive correlation between the strengths of each item's context-to-item association and its item-to-item associations with other items with which it was rehearsed. Subjects in the free-recall condition begin recall by accessing those items with the strongest contextto-item associations and, by necessity, the strongest associations to other list items. Since these firstrecalled items are then used as "cues" for the recall of other items, free-recall subjects begin recall with the best available cues, whereas subjects in the cued-recall condition begin recall with experimenter-provided cues, some of which may be poor cues. Moreover, since experimenter-provided cues direct cued-recall subjects to clusters of items that were associated with one another during study, the clusters with which cued-recall subjects begin their recall are, on the average, less rich in critical items (because these clusters necessarily contain the cues themselves) than are those clusters with which freerecall subjects begin their recall. (The clusters with which free-recall subjects begin recall could contain only critical items and no "cues.") Thus, performance in the free-recall condition is superior to that in the cued-recall condition. However, this explanation cannot be easily applied to semantic memory paradigms because (1) semantic memory experiments typically do not involve a prior study of the items that would produce a positive correlation between the strengths of an item's contextto-item and item-to-item associations and (2) the cluster to which the search is directed by the category-nameplus-letter probe in the related and unrelated priming conditions is equally rich in "critical" items. Because previous applications of SAM have used different mechanisms to account for inhibition from semantically related primes in episodic and semantic memory, they are somewhat unparsimonious.

A second general approach for accommodating the inhibitory priming effects obtained in the present experiments is embodied in a model of attention proposed by Keele and Neill (1978). In their model, a stimulus activates its own representation in memory, and this activation spreads to related nodes, thereby producing facilitation such as that observed in the related priming conditions in our read-generate and read-read groups. However, this spreading activation ultimately leads to inhibition effects because the activation in featurally and semantically similar representations must be actively suppressed if the stimulus that produced the activation is to be precisely identified. Thus, on related priming trials in which the prime comes from the same category as the target, the target response is activated along with the priming response and hence must be directly suppressed so that the correct priming response may be given. Since the target response would not have been activated, and hence would not have been suppressed, on an unrelated priming trial, a target would be easier to generate or name following unrelated priming trials, on which it had not been suppressed, than following related priming trials, on which it had been suppressed. Although the SAM model and an account based on Keele and Neill's theory explain the present findings in very different ways-by prior activation of the priming items blocking target retrieval versus prior suppression of the target making it less accessible-we believe that each of these models will, without further specification, have trouble predicting exactly when related primes will produce facilitation or inhibition (but see Neill, 1977, 1979, for some preliminary specifications of the Keele-Neill model).

A third way of handling both facilitation and inhibition from semantically related primes is to assume that there are both excitatory and inhibitory connections between related nodes in the memory network (see Martindale, 1981, and Walley \& Weiden, 1973). According to this view, inhibitory priming effects are similar to lateral inhibition effects that occur in the visual sensory system (e.g., Ratliff, 1965). But once again this theoretical framework must be elaborated with precise rules that specify how the excitation and inhibition combine to determine whether priming produces a net facilitation or inhibition effect.

In closing, it is important to note that the inhibitory semantic priming effects reported here and in A.S. Brown (1981) and A.S. Brown et al. (Note 1) are not confined to variations of the Freedman-Loftus paradigm. Evidence of inhibition from semantically related primes has also been reported in episodic recall (see 
previous references in our discussion of SAM) and episodic recognition tasks (e.g., Neely, Schmidt, \& Roediger, 1983; Todres \& Watkins, 1981), in perceptual identification (e.g., Underwood, 1981) and matching tasks (Neill, 1979), and under certain conditions in a category matching task (Rosch, 1975). Until there is direct evidence that the inhibition effects in these diverse paradigms are produced by different mechanisms, we feel that, for parsimony's sake, a single integrative theory should be sought. Exactly what form this theory will take is unclear. However, it is clear that the challenge for such a theory is to specify in advance the conditions under which semantically related primes will facilitate or inhibit performance in various perceptual and memory tasks.

\section{REFERENCE NOTE}

1. Brown, A. S., Bean, A. D., \& Whiteman, S. L. Decisional versus retrieval factors in semantic prime interference. Unpublished manuscript, 1982.

\section{REFERENCES}

Anderson, J. R. Language, memory, and thought. Hillsdale, N.J: Erlbaum, 1976.

Balota, D. A. Automatic semantic activation and episodic memory encoding. Journal of Verbal Learning and Verbal Behavior, 1983, 22, 88-104.

Battig, W. F., \& Montague, W. E. Category norms for verbal items in 56 categories: A replication and extension of the Connecticut category norms. Journal of Experimental Psychology Monograph, 1969, 80(3, Pt. 2).

Becken, C. A. Semantic context and word frequency effects in visual word recognition. Journal of Experimental Psychology: Human Perception and Performance, 1979, 5, 252-259.

BeCKer, C. A. Semantic context effects in visual word recognition: An analysis of semantic strategies. Memory \& Cognition, $1980,8,493-512$.

Brown, A. S. Priming effects in semantic memory retrieval processes. Journal of Experimental Psychology: Human Learning and Memory, 1979, 5, 65-77.

Brown, A. S. Inhibition in cued retrieval. Journal of Experimental Psychology: Human Learning and Memory, 1981, 7, 204-215.

Brown, J. Reciprocal facilitation and impairment of free recall. Psychonomic Science, 1968, 10, 41-42.

Carr, T. H., McCauley, C., Spereer, R. D., \& Parmelee, C. M. Words, pictures, and priming: On semantic activation, conscious identification, and the automaticity of information processing. Journal of Experimental Psychology: Human Perception and Performance, 1982, 8, 757-777.

Collins, A. M., \& Loftus, E. F. A spreading activation theory of semantic processing. Psychological Review, 1975, 82, 407-428.

Fischler, I. Semantic facilitation without association in a lexical decision task. Memory \& Cognition, 1977, 5, 335-339.

Fischlen, I., \& Goodman, G. O. Latency of associative activation in memory. Journal of Experimental Psychology: Human Perception and Performance, 1978, 4, 455-470.

Fowlen, C. A., Wolford, G., Slade, R., \& Tassinary, L. Lexical access with and without awareness. Journal of Experimental Psychology: General, 1981, 110, 341-362.

Freedman, J. L., \& Loftus, E. F. Retrieval of words from long term memory. Journal of Verbal Learning and Verbal Behavior, $1971,10,107-115$.

Henik, A., Friedrich, F. J., \& Kelloga, W. A. The dependence of semantic relatedness effects upon prime processing. Memory \& Cognition, 1983, 11, 366-373.
Hunt, K. P., \& Hodge, M. H. Category-item frequency and category-name meaningfulness $\left(\mathrm{m}^{\prime}\right)$ : Taxonomic norms for 84 categories. Psychonomic Monograph Supplements, 1971, 4, 97-121.

IRWIN, D. I., \& LUPKER, S. J. Semantic priming of pictures and words: A levels of processing approach. Journal of Verbal Learning and Verbal Behavior, 1983, 22, 45-60.

KARCHMER, M. A., \& Winograd, E. Effects of studying a subset of familiar items on recall of the remaining items: The John Brown effect. Psychonomic Science, 1971, 25, 224-225.

KeELE, S. W., \& Neill, W. T. Mechanisms of attention. In E. C. Carterette \& P. Friedman (Eds.), Handbook of perception (Vol. 9). New York: Academic Press, 1978.

LofTus, E. F. Activation of semantic memory. American Journal of Psychology, 1973, 86, 331-337.

Loftus, G. R., \& Loftus, E. F. The influence of one memory retrieval on a subsequent memory retrieval. Memory \& Cognition, 1974, 2, 467-471.

LoRch, R. F. Priming and search processes in semantic memory: A test of three models of spreading activation. Journal of Verbal Learning and Verbal Behavior, 1982, 3, 468-492.

MARCEL, A. J. Conscious and preconscious recognition of polysemous words: Locating the selective effects of prior verbal context. In R. S. Nickerson (Ed.), Attention and performance VIII. Hillsdale, N.J: Erlbaum, 1980.

Marcel, A. J., \& Patterson, K. E. Word recognition and production: Reciprocity in clinical and normal studies. In J. Requin (Ed.), Attention and performance VII. Hillsdale, N.J: Erlbaum, 1978.

Martindale, C. Cognition and consciousness. Homewood, Ill: Dorsey Press, 1981.

McCauley, C., Pahmelee, C. M., Sperber, R. D., \& Carr, T. H. Early extraction of meaning from pictures and its relation to conscious identification. Journal of Experimental Psychology: Human Perception and Performance, 1980, 6, 265-276.

MerikLe, P. M. Unconscious perception revisited. Perception \& Psychophysics, 1982, 31, 298-301.

Meyer, D. E., \& Schvaneveldt, R. W. Facilitation in recognizing pairs of words: Evidence of a dependence between retrieval operations. Journal of Experimental Psychology, 1971, 90, 227-234.

Meyer, D. E., Schvaneveldt, R. W., \& Ruddy, M. G. Loci of contextual effects on visual word recognition. In P. M. A. Rabbitt \& S. Dornic (Eds.), Attention and performance $V$. New York: Academic Press, 1975.

Muelle R, C. W., \& Watkins, M. J. Inhibition from part-set cueing: A cue-overload interpretation. Journal of Verbal Learning and Verbal Behavior, 1977, 16, 699-710.

NEELY, J. H. Semantic priming and retrieval from lexical memory: Evidence for facilitatory and inhibitory processes. Memory \& Cognition, 1976, 4, 648-654.

NeEly, J. H. Semantic priming and retrieval from lexical memory: Roles of inhibitionless spreading activation and limited capacity attention. Journal of Experimental Psychology: General, 1977, 106, 226-254.

Neely, J. H., Schmidt, S. R., \& Roediger, H. L. Inhibition from related primes in recognition memory. Journal of Experimental Psychology: Learning, Memory, and Cognition, 1983, 9, 196-211.

Ne ILL, W. T. Inhibitory and facilitatory processes in selective attention. Journal of Experimental Psychology: Human Perception and Performance, 1977, 3, 444-450.

NeILL, W. T. Switching attention within and between categories: Evidence for intracategory inhibition. Memory \& Cognition, $1979,7,283-290$.

Parkin, A. J. Specifying levels of processing. Quarterly Journal of Experimental Psychology, 1979, 31, 179-195.

Posner, M. I., \& SNyder, C. R. R. Attention and cognitive control. In R. L. Solso (Ed.), Information processing and cognition: The Loyola symposium. Hillsdale, N.J: Erlbaum, 1975.

Potter, M. C., \& Faulconer, B. A. Time to understand pictures and words. Nature, 1975, 253, 437-438. 
RaAijmakers, J. G., \& Shiffain, R. M. Search of associative memory. Psychological Review, 1981, 88, 93-134.

RAtcliff, R., \& McKoon, G. Does activation really spread? Psychological Review, 1981, 88, 454-462.

RATLIFF, F. Mach bands: Quantitative studies on neural networks in the retina. San Francisco: Holden-Day, 1965.

ROEDIGer, H. L. Inhibition in recall from cueing with recall targets. Journal of Verbal Learning and Verbal Behavior, 1973, 12, 644-657.

Roediger, H. L., \& Neelx, J. H. Retrieval blocks in episodic and semantic memory. Canadian Journal of Psychology, 1982, 36, 213-242.

Roediger, H. L., Neely, J. H., \& Blaxton, T. A. Inhibition from related primes in semantic memory retrieval: A reappraisal of Brown's (1979) paradigm. Journal of Experimental Psychology: Learning, Memory, and Cognition, 1983, 9, 478-485.

Roediger, H. L., \& Schmidt, S. R. Output interference in the recall of categorized and paired associate lists. Journal of Experimental Psychology: Human Learning and Memory, 1980, 6, 91-105.

Roediger, H. L., \& Tulving, E. Exclusion of material from recall as a post-retrieval operation. Journal of Verbal Learning and Verbal Behavior, 1979, 18, 601-615.

Rosch, E. Cognitive representations of semantic categories. Journal of Experimental Psychology: General, 1975, 104, 192-233.

Rundus, D. Negative effects of using list items as recall cues. Journal of Verbal Learning and Verbal Behavior, 1973, 12, 43-50.

Schvaneveldt, R. W., \& MEYeR, D. E. Retrieval and comparison processes in semantic memory. In S. Kornblum (Ed.), Attention and performance IV. New York: Academic Press, 1973.

Shap1ro, S. I., \& Palermo, D. S. Conceptual organization and class membership: Normative data for representatives of 100 categories. Psychonomic Monograph Supplements, 1970, 3, 107-127.

Sмiтh, A. D. Output interference and organized recall from longterm memory. Journal of Verbal Learning and Verbal Behavior, $1971,10,400-408$.

Sмiтh, A. D. Input order and output interference in organized recall. Journal of Experimental Psychology, 1973, 100, 147-150.

Sмiтh, M. C. Contextual facilitation in a letter search task depends on how the prime is processed. Journal of Experimental Psychology: Human Perception and Performance, 1979, 5, 239-251.

Smith, M. C., Theodor, L., \& Franklin, P. E. The relationship between contextual facilitation and depth of processing. Journal of Experimental Psychology: Learning, Memory, and Cognition, 1983, 9, 697-712.

Todres, A. K., \& Watkıns, M. J. A part-set cuing effect in recognition. Journal of Experimental Psychology: Human Learning and Memory, 1981, 7,91-99.

UNDE RWOOD, G. Lexical recognition of embedded unattended words: Some implications for reading processes. Acta Psychologica, 1981, 47, 267-283.

Walley, R. E., \& Weiden, T. D. Lateral inhibition and cognitive masking: A neuropsychological theory of attention. Psychological Review, 1973, 80, 284-302.
WatKıns, M. J. Inhibition in recall with extralist "cues." Journal of Verbal Learning and Verbal Behavior, 1975, 14, 294-303.

\section{NOTES}

1. According to this analysis, obtaining larger inhibition effects with an increase in the number of prior tests on the currently tested category is all the more compelling, since with a greater number of previous related priming trials, the more likely it is that the current target itself will have previously been retrieved on those priming trials. In most current theories, these previous retrievals of the target presumably would facilitate, rather than inhibit, its retrieval. (But see our discussion of the Keele \& Neill, 1978, theory in the General Discussion section.)

2. It must be acknowledged that A. S. Brown (1981, Experiment 5) conducted a control experiment to rule out an explanation of his data based on a "general fatigue" effect. However, it was an inadequate control, because it differed from Brown's other inhibition-producing experiments in that subjects were never tested on more than one item from any particular semantic category. Of course, it might be argued that such a control experiment was unnecessary because A. S. Brown's (1981) Experiment 1 already contained an implicit unrelated control priming condition. That is, the fifth test on a category followed only four consecutive priming tests on a single category (i.e., the same category as the target's category), whereas the first test on a category followed five consecutive tests on a single category (i.e., a category unrelated to the target's category). This line of reasoning implies that since Brown found slower response times to the fifth test on a category than to the first test on a category, his results do indeed demonstrate category-specific inhibition. However, since the first test on a category was preceded by the 5 -sec warning presentation of a category name, whereas the fifth test was not, it is possible that during this 5 -sec period there was a dissipation of nonspecific inhibition that had accrued during the five prior tests on the single semantic category unrelated to the target's category. If such a dissipation occurred, Brown's inhibition effect may have been due to nonspecific inhibition rather than to category-specific inhibition.

3 . There is one set of findings that runs counter to the conclusion that an experimenter-provided prime must be processed to a deep level if semantic priming is to occur. Several studies have shown that semantic priming can occur under conditions in which subjects cannot even detect the presence of the prime (Balota, 1983; Fowler, Wolford, Slade, \& Tassinary, 1981; Marcel, 1980; Marcel \& Patterson, 1978; but see also Merikle, 1982) or cannot identify the prime (Carr, McCauley, Sperber, \& Parmelee, 1982; Fischler \& Goodman, 1978; McCauley, Parmelee, Sperber, \& Carr, 1980). Apparently, then, the depth-of-processing effect on priming occurs only for consciously encoded primes and not for primes presented outside conscious awareness.

(Manuscript received July 18,1982 ; revision accepted for publication July 23,1983 .) 